H I G H L I G H T S

IN BRIEF

\section{SIGNAL TRANSDUCTION}

Ras regulates assembly of mitogenic signaling complexes through the effector protein IMP.

Matheny, S. A. et al. Nature 427, 256-260 (2004)

The Ras signalling cascade includes Raf, mitogen-activated protein (MAP) kinase and MAP kinase kinase (MEK). Matheny et al. have now identified IMP, a Ras effector that negatively regulates MAP-kinase activation. IMP limits the formation of Raf-MEK complexes in the absence of Ras activation. Ras signalling can inactivate IMP through auto-polyubiquitylation, which releases the inhibition of complex formation.

\section{REPLICATION}

Identification of two origins of replication in the single chromosome of the archaeon Sulfolobus solfataricus.

Robinson, N. P. et al. Cell 116, 25-38 (2004)

Archaeons have a minimal replication apparatus that resembles that of eukaryotes. Robinson et al. now show that archaeons, like eukaryotes, use multiple origins of replication. S. solfataricus has two active origins, which are recognized selectively by its three homologues of the eukaryotic initiator proteins Cdc6 and Orc1.

\section{DEVELOPMENT}

Cup is an elF4E binding protein required for both the translational repression of oskar and the recruitment of Barentsz.

Wilhelm, J. E. et al. J. Cell Biol. 163, 1197-1204 (2003)

Drosophila Cup is an elF4A binding protein that associates with Bruno and regulates oskar mRNA translation in oogenesis.

Nakamura, A. et al. Dev. Cell 6, 69-78 (2004)

In fly oogenesis, oskar mRNA is translationally repressed until it is correctly localized to the posterior pole. Two groups have now identified Cup, a translation initiation factor $4 \mathrm{E}$ (eIF4E)-binding protein, as a translational repressor of oskar. Wilhelm et al. also show that Cup is required to recruit the localization factor Barentsz to the oskar ribonucleoprotein complex, thereby providing a likely regulatory link between localization and translation.

\section{TECHNIQUES}

\section{Detection of molecular interactions at membrane} surfaces through colloid phase transitions.

\section{Baksh, M. M. et al. Nature 427, 139-141 (2004)}

Studies of biochemical events at membrane surfaces are technically challenging. Using lipid membranes supported on a silica substrate, Baksh et al. now suggest an experimental approach that involves adjusting the lipid membrane composition so that the membrane system is close to a phase transition. Small perturbations on the membrane surface, such as molecular interactions, induce easily measurable changes in the macroscopic organization of the colloid.

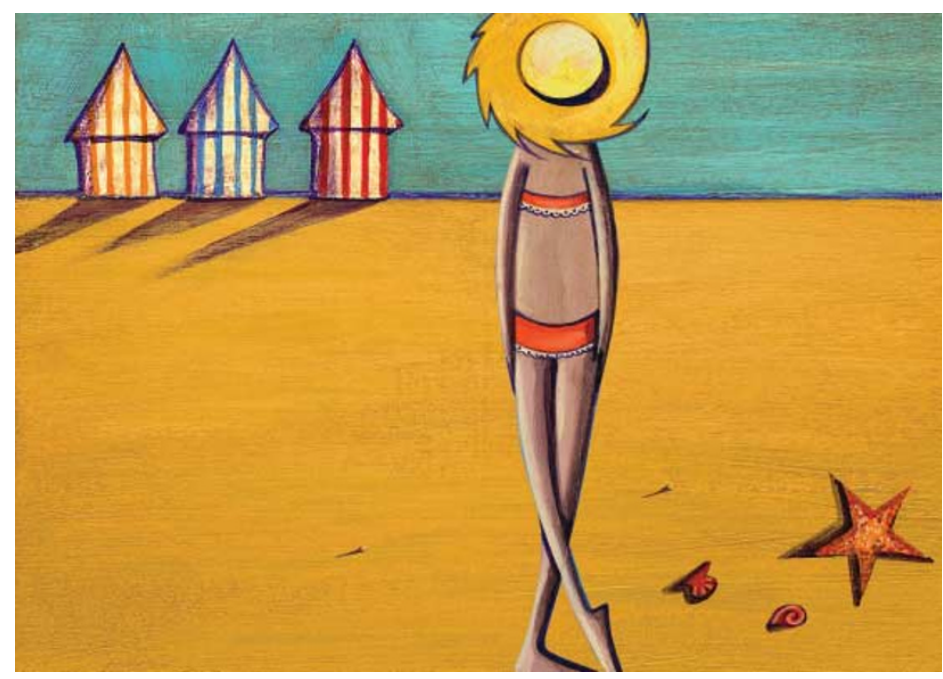

CHROMATIN

\section{Aiming for some peace and quiet}

The RNA interference (RNAi) pathway has been implicated in heterochomatin assembly and gene silencing in fission yeast. And now, Grewal, Moazed and colleagues have identified a complex — RITS (RNA-induced initiation of transcriptional gene silencing) — that provides a mechanistic basis for RNAi-mediated targeting of heterochromatin.

Reporting in Science, the authors tested whether Chpl - a heterochromatin-associated protein that binds centromeric repeats and is required for the heterochromatin-specific methylation of histone $\mathrm{H} 3$ at lysine 9 (H3-K9) and the recruitment of the heterochromatin-binding protein Swi6 - might provide the link between RNAi and heterochromatin. They affinity-purified extracts from a yeast strain that produces tagged Chp1 and, in addition to Chp1, identified Ago1, an Argonaute family protein, and an unknown protein, which they named Tas3 (targeting complex subunit 3 ). The purification profile was identical when tagged Tas 3 was used instead, which confirmed that Chp1, Tas3 and Agol form a complex - RITS.

Chp1 and Agol are required for heterochromatin assembly and gene silencing within heterochromatic regions. Grewal, Moazed and co-workers wanted to know whether Tas 3 has similar properties, which it indeed has, as a tas 3 deletion strain carrying a reporter gene that was inserted in centromeric repeats showed loss of gene silencing. And chromatin immunoprecipitation (ChIP) studies showed that, in tas $3 \Delta$ cells, H3-K9 methylation and recruitment of Swi6 to the reporter gene were abolished.

Next, the authors found that the RITS complex was associated with small RNA molecules of 22-25 nucleotides. However, these small RNA species were absent from RITS that was purified from a strain lacking Dcr1, the fission yeast homologue of the Dicer enzyme, which generates small interfering RNA (siRNA). So, the RITS-associated small RNAs require Dcr1 for their production. Moreover, northern- and Southern-blot analyses established that the siRNAs that are associated with RITS originate from centromeric repeat sequences - providing RITS with target specificity.

To determine the in vivo chromatin localization of RITS, the authors carried out ChIP experiments, which showed that tagged Tas 3 localizes to centromeric repeats in wild-type cells, but not in cells that lacked factors involved in the RNAi pathway (Agol, Dcr1 or RNA-dependent RNA polymerase). This indicates the direct requirement of the RNAi pathway for the targeting of heterochomatin by RITS. The authors propose that RITS uses Dcr1-produced siRNAs to recognize and bind specific chromosome regions to initiate heterochromatin assembly and gene silencing.

\section{4) References and links}

Arianne Heinrichs

ORIGINAL RESEARCH PAPER Verdel, A. et al. RNAi-mediated targeting of heterochromatin by the RITS complex. Science 2 Jan 2004 (doi:10.1126/science.1093686) 